\title{
(In)segurança alimentar e nutricional em famílias do município de Gramados dos Loureiros (RS) e a percepção acerca da atuação do profissional Nutricionista
}

\section{Food and nutritional (in)security in families in the municpality of Gramados dos Loureiros (RS) and the perception about the performance of the profissional Nutririonist}

\author{
Bianca Gabrieli Silva, Vanessa Girotto, Nádia Kunkel Szinwelski \\ e Junir Antônio Lutinski
}

Como citar este artigo: SILVA, BIANCA G.; CIROTTO, VANESSA.; SZINUELSKI, NÁDIA K.; LUTINSKI, JUNIR A. (In)segurança alimentar e nutricional em familias do município de Gramados dos Loureiros (RS) e a percepção acerca da atuação do profissional Nutricionista. Revista Saúde (Sta. Maria). 2021; 47.

\section{Autor correspondente: \\ Nome: Junir Antonio Lutinski \\ E-mail: junir@unochapeco.edu.br \\ Telefone: (49) 3231-8215 \\ Formação Profissional: Biólogo, \\ Dr. em Biodiversidade Animal. Pro- fessor do Programa de Pós-Gra- duação em Ciências da Saúde da Universidade Comunitária da Região de Chapecó, Chapecó, Santa Catarina.}

Filiação Institucional: Programa de Pós-Graduação em Ciências da Saúde da Universidade Comunitária da Região de Chapecó, Chapecó, Santa Catarina, Brasil. Endereço para correspondência: Rua Beija-Flor, 254 E

Bairro: Efapi

Cidade: Chapecó

Estado: Santa Catarina

CEP: $89809-760$

Data de Submissão:

04/09/2020

Data de aceite:

04/02/2021

Conflito de Interesse: Não há conflito de interesse

\section{(cc) $\mathrm{BY}-\mathrm{NC}-\mathrm{ND}$}

\section{RESUMO:}

Introdução: A insegurança alimentar permeia o cotidiano de uma parcela crescente da população. 0 profissional Nutricionista atua na área da saúde orientando pacientes de forma privada ou coletiva para ter uma alimentação adequada. Objetivo: 0 estudo teve com objetivo avaliar o estado (in) segurança alimentar das famílias do município de Gramado dos Loureiros, RS bem como identificar a percepção das mesmas sobre o trabalho do profissional Nutricionista, descrevendo a frequência e a preferência alimentar dos indivíduos entrevistados (chefes de Famílias maiores de 18 anos). Metodologia: $O$ estudo foi realizado entre janeiro e março de 2020. Foram utilizados questionários semiestruturados, aplicados nas zonas urbana e rural. Resultados: Observou-se que a maioria (54\%) das famílias se encontra em algum nível de insegurança alimentar. Os entrevistados da zona rural apresentaram melhores resultados em relação à segurança alimentar. A maioria (93\%) demonstrou ter conhecimento e se importar com a qualidade dos alimentos consumidos. A maioria (82\%) já consultou ou teve um membro da família atendido por um profissional Nutricionista. Ao todo, $85 \%$ acreditam que o profissional Nutricionista agrega qualidade ao serviço de saúde no município. Conclusão: $A$ insegurança alimentar está presente nas famílias gramadenses. A população tem acesso e interesse a uma alimentação adequada, contudo, o sentimento de medo e incertezas do amanhã ainda é um fator preocupante.

PALAVRAS-CHAVE: Atenção primária em saúde; Determinantes sociais da saúde; Promoção da saúde; Segurança Alimentar e Nutricional.

\section{ABSTRACT}

Introduction: Food insecurity permeates an increasing portion of the population. The Nutritionist professional works in the health area, guiding patients privately or collectively to have an adequate diet. Objective: The study aimed to assess the food (in)security status of families in the municipality of Gramado dos Loureiros, RS, as well as to identify their perception of the work of the professional Nutritionist, describing the frequency and food preference of the interviewed individuals (families heads over 18). Methodology: The study was carried out between January and March 2020. Semi-structured questionnaires were used, applied in urban and rural areas. Results: It was observed that the majority $(54 \%)$ of families are at some level of food insecurity. Respondents from the countryside showed better results in terms of food security. The majority (93\%) demonstrated knowledge and care about the quality of the food consumed. The majority $(82 \%)$ have already consulted or had a family member attended by a professional Nutritionist. In all, $85 \%$ believe that the professional Nutritionist adds quality to the health service in the municipality. Conclusion: Food insecurity is present in the families of Gramados dos Loureiros. The population has access and interest in adequate food, however, the feeling of fear and uncertainty of tomorrow is still a worrying factor.

KEYWORDS: Primary health care; Social determinants of health; Health promotion; Food and nutrition security. 


\section{INTRODUÇÃO}

O conceito de segurança alimentar (SAN) começou a ser tratado a partir do início do século XX. No Brasil, na década de 1930, a alimentação e a nutrição começaram a ser discutidas como uma questão de política pública. A partir de 1996 a SAN passou a ser compreendida como uma estratégia para garantir o Direito Humano à Alimentação Adequada (DHAA) 1 .

Em 2006, entrou em vigor a Lei Orgânica de Segurança Alimentar e Nutricional (LOSAN), que visa assegurar o direito humano à alimentação adequada², que estabelece no Art. $2^{\circ}$ "A alimentação adequada é direito fundamental do ser humano, inerente à dignidade da pessoa humana e indispensável à realização dos direitos consagrados na Constituição Federal, devendo o poder público adotar as políticas e ações que se façam necessárias para promover e garantir a segurança alimentar e nutricional da população".2

A LOSAN descreve a Segurança Alimentar e Nutricional (SAN) como "a estratégia ou conjunto de ações, deve ser intersetorial e participativa, e consiste na realização do direito de todos ao acesso regular e permanente a alimentos de qualidade, em quantidade suficiente, sem comprometer o acesso a outras necessidades essenciais, tendo como base práticas alimentares promotoras da saúde, que respeitem a diversidade cultural e que sejam ambiental, cultural, econômica e socialmente sustentáveis." ${ }^{2}$

Percebe-se que o conceito aborda dois elementos distintos e complementares, a dimensão alimentar refere-se à produção e disponibilidade de alimentos; e a dimensão nutricional incorpora as relações entre o ser humano e o alimento. Assim, a garantia do alimento adequado em proporções seguras, traz consigo a validação nutricional apropriada às fases de vida, assegurando o pleno crescimento, desenvolvimento e qualidade de vida benemerente ${ }^{3}$. Determinado indivíduo está em estado de Segurança Alimentar e Nutricional, quando tem acesso regular a alimentação e nutrição adequadas e tem plenas condições de aproveitar, em termos fisiológicos, os alimentos ingeridos¹.

Dados do Instituto Brasileiro de Geografia e Estatística mostram que, em uma comparação internacional com países que seguem metodologia semelhante, em 2013, o Brasil apresentava uma prevalência de segurança alimentar acima dos demais países latino-americanos. Contudo, no mundo, a Organização das Nações Unidas para Alimentação e a Agricultura (FAO) estimou que 815 milhões de pessoas em 2016 e quase 821 milhões em $2017^{5}$ passavam fome. Oscilações na economia como a desencadeada pela pandemia de COVID 19 afetam a segurança alimentar e nutricional da população ${ }^{6}$.

Existem diversos métodos que podem ser utilizados para medir a Insegurança Alimentar e Nutricional - INSAN, sendo que cada um capta o fenômeno segundo uma escala e uma ótica própria. Enquanto alguns métodos avaliam os fatores determinantes, como a ingestão alimentar e os gastos com a alimentação, outros avaliam as consequências da 
insegurança alimentar, como a antropometria. A avaliação da segurança alimentar deve ser baseada na aplicação de vários destes métodos, permitindo que diferentes dimensões do problema possam ser avaliadas ${ }^{3}$.

O profissional Nutricionista atua orientando pacientes de forma privada ou coletiva visando uma alimentação adequada. Ele é capacitado para cuidar do equilíbrio alimentar e nutricional, com ênfase na boa qualidade de vida e no desempenho saudável. É responsável por promover, prevenir e orientar agravos consequentes de uma má alimentação. Sua atuação é primordial na promoção da qualidade de vida e saúde das pessoas, refletindo na disposição e na capacidade de realizar atividades do cotidiano ${ }^{7}$.

Os motivos que levam uma pessoa a optar por determinado alimento são relevantes para que se entenda a situação alimentar do indivíduo, seja ela de segurança ou insegurança alimentar. $\mathrm{O}$ ato de se alimentar e nutrir engloba aspectos fisiológicos, econômicos, psicológicos, culturais e socioeconômicos, como a escolaridade, renda, disponibilidade de alimentos e preço ${ }^{8}$.

As motivações alimentares levam o indivíduo a escolher o que ele vai comer, se vai comer ou quando poderá comer. Os hábitos alimentares dos indivíduos são totalmente desiguais uns dos outros, sendo assim cada pessoa tornase especial, ou seja, perante um profissional da saúde é necessário utilizar-se de técnicas e estratégias diferentes para cada pessoa, assim como para cada fase da vida'. 0 acesso de uma família a um profissional Nutricionista tem sido maior graças a programas como a Estratégia de saúde da Família (ESF), junto ao Núcleo de Apoio à Saúde da Família $(\mathrm{NASF})^{9}$.

Neste contexto, emerge a seguinte pergunta de estudo: residir em um município de pequeno porte confere segurança alimentar e acesso a serviço nutricional especializado? Assim, este estudo teve como objetivos a) avaliar 0 estado de (in) segurança alimentar e nutricional de Famílias do município de Gramado dos Loureiros (RS); b) descrever a frequência e a preferência alimentar de uma amostra dos chefes de família do município e c) identificar a percepção de chefes de famílias acerca da atuação do profissional Nutricionista.

\section{MÉTODO}

A pesquisa foi aprovada pelo CONEP/CEP sob parecer 3.771.671 e se caracterizou como quantitativa e de caráter transversal ${ }^{10}$. Foi realizada no município de Gramado dos Loureiros, RS, localizado na intersecção das coordenadas de latitude $27^{\circ} 26^{\prime} 38^{\prime \prime}$ sul e de longitude $52^{\circ} 55^{\prime} 03^{\prime \prime}$ oeste que possui área territorial de $131,396 \mathrm{Km}^{2}$. A taxa de escolaridade é de 99,5\% na faixa etária entre 6 a 14 anos. 0 índice de desenvolvimento humano (IDH) do município é de 0,685. A população estimada em 2019 era de 2.082 pessoas. Em 2017, o salário médio mensal era de 0,9 salários mínimos. 0 PIB per capita é de $R \$ 19.704,15$. A faixa etária que predomina o município é de 10 a 14 anos ${ }^{11}$. 
Participaram cidadãos residentes no município, acima de 18 anos, homens ou mulheres, que se identificam como chefes da família e que concordaram formalmente em participar da pesquisa, mediante assinatura de Termo de Consentimento Livre e Esclarecido. Foi utilizada a técnica de snowball para compor a amostra ${ }^{12}$. A pesquisa foi realizada entre os meses de janeiro e março de 2020 nos próprios domicílios dos participantes.

Para avaliar o nível de (in)segurança alimentar e nutricional foi utilizado o questionário da escala Brasileira de Insegurança Alimentar (EBIA). Esse método demonstra os níveis de insegurança ou de segurança alimentar. A EBIAé um parâmetro utilizado para entender a dificuldade de acesso familiar aos alimentos e também às dimensões psicológicas e sociais da insegurança alimentar. Essa escala é descrita em quatro categorias: a de segurança alimentar se confere aos domicílios que possuem acesso regular e permanente a alimentos em quantidades suficientes e de qualidade adequada. A insegurança alimentar pode ser dividida em leve, moderada e grave ${ }^{13,14 .}$

Para verificar a frequência e a preferência alimentar das famílias, foi utilizado o questionário de frequência alimentar do SISVAN juntamente com um recordatório alimentar dos últimos sete dias do entrevistado, proposto pelo Ministério da Saúde. Esse recordatório conta com questões objetivas referentes à alimentação correspondente à semana anterior. Assim, informa quantas vezes, na última semana, o participante ingeriu determinado grupo alimentar ${ }^{2,7}$. 0 questionário de frequência alimentar (QFA) permite ao entrevistador questionar sobre o consumo de alimentos ingeridos pelo entrevistado durante um longo período de tempo. Trata-se de um instrumento que apresenta uma lista de alimentos e preparações com porções padronizadas, solicitando saber qual a quantidade ingerida em um determinado período (dias ou semanas) $)^{2}$.

Para identificar a percepção dos participantes quanto à atuação do profissional Nutricionista foi aplicado um questionário semiestruturado contendo 10 questões objetivas. Esse questionário abordou questões sobre a alimentação, a profissão Nutricionista, bem como histórico de uso dos serviços desse profissional para si ou para familiares, assim como doenças relacionadas aos hábitos e preferências alimentares como diabetes mellitus (DM), hipertensão arterial sistêmica (HAS) e dislipidemias ${ }^{15}$.

Os dados foram tabulados em um banco de dados utilizando o Software Excel for Windows. Foram realizadas análises exploratórias baseadas nas frequências, medidas de tendência central, medidas de dispersão e ilustradas em tabelas e gráficos. Para os instrumentos já validados (Frequência Alimentar e EBIA) foram adotadas as classificações já padronizadas disponíveis na literatura para cada caso ${ }^{14}$.

\section{RESULTADOS}

Ao todo, 46 munícipes gramadenses participaram da pesquisa. 0 sexo feminino foi o mais frequente, assim 
como prestadores de serviço, seguido de vendedores e agricultores. A maioria dos participantes do público feminino relatou já ter consultado com um Nutricionista. $\mathrm{O}$ acesso ao Nutricionista foi maior na zona urbana em relação à zona rural (Tabela 1).

Tabela 1: Perfil dos participantes do estudo residente no município de Gramados dos Loureiros, RS, janeiro a março de 2020.

\begin{tabular}{|c|c|c|c|c|}
\hline \multirow[b]{2}{*}{ Variável } & \multicolumn{2}{|c|}{ Total da amostra } & \multicolumn{2}{|c|}{ Consulta a Nutricionista } \\
\hline & Contagem & Percentual & Contagem & Percentual \\
\hline \multicolumn{5}{|l|}{ Sexo } \\
\hline Feminino & 25 & 54,3 & 21 & 84,0 \\
\hline Masculino & 21 & 45,7 & 17 & 81,0 \\
\hline \multicolumn{5}{|l|}{ Zona de residência } \\
\hline Rural & 23 & 50,0 & 17 & 73,9 \\
\hline Urbana & 23 & 50,0 & 21 & 91,3 \\
\hline \multicolumn{5}{|l|}{ Ocupação } \\
\hline Agricultor & 8 & 17,4 & 5 & 62,5 \\
\hline Aposentado & 4 & 8,7 & 4 & 100,0 \\
\hline Auxiliar administrativo & 3 & 6,5 & 3 & 100,0 \\
\hline Dona de casa & 2 & 4,3 & 1 & 50,0 \\
\hline Estudante & 4 & 8,7 & 4 & 100,0 \\
\hline Motorista & 1 & 2,2 & 0 & 0,0 \\
\hline Prestador de serviços & 10 & 21,7 & 9 & 90,0 \\
\hline Professor & 2 & 4,3 & 1 & 50,0 \\
\hline Servidor público & 3 & 6,5 & 3 & 100,0 \\
\hline Vendas & 9 & 19,6 & 8 & 88,9 \\
\hline
\end{tabular}

Menos da metade das famílias encontram-se em segurança alimentar. Os adultos apresentaram uma maior pontuação em relação à classificação de insegurança grave (Tabela 2). Das famílias residentes na zona rural do município, a maioria apresentou segurança alimentar, seguindo de insegurança moderada. Já na área urbana, a maioria demonstrou insegurança alimentar leve, seguindo de segurança. Participantes do sexo feminino apresentaram mais segurança alimentar em relação ao masculino (Tabela 3). 
Tabela 2: Classificação de (in)segurança alimentar entre os participantes da pesquisa, município de Gramados dos Loureiros, RS, janeiro a março de 2020. n: número de participantes.

\begin{tabular}{lccccc}
\hline Classificação EBIA & $\mathbf{n}$ & Pontos adultos & $\begin{array}{c}\text { Pontos menores } \\
\text { de } 18 \text { anos }\end{array}$ & Soma dos pontos & Percentual \\
\hline Leve & 13 & 20 & 8 & 28 & 28,3 \\
Moderada & 6 & 22 & 5 & 27 & 13,0 \\
Grave & 6 & 28 & 12 & 40 & 13,0 \\
Segurança & 21 & 0 & 0 & 0 & 45,7 \\
\hline
\end{tabular}

Tabela 3: Classificação da (in)segurança alimentar segundo sexo e zona residência dos participantes da pesquisa, município de Gramados dos Loureiros, janeiro a março de 2020. n: número de participantes.

\begin{tabular}{lcccccccc}
\hline \multirow{2}{*}{ Variável } & \multicolumn{2}{c}{ Leve } & \multicolumn{2}{c}{ Moderada } & \multicolumn{2}{c}{ Grave } & \multicolumn{2}{c}{ Segurança } \\
& $\mathrm{N}$ & $\%$ & $\mathrm{~N}$ & $\%$ & $\mathrm{n}$ & $\%$ & $\mathrm{n}$ & $\%$ \\
\hline Sexo & & & & & & & & \\
Feminino & 6 & 24,0 & 4 & 16,0 & 3 & 12,0 & 12 & 48,0 \\
Masculino & 7 & 33,3 & 2 & 9,5 & 3 & 14,3 & 9 & 42,9 \\
Zona de residência & & & & & & & & \\
Rural & 2 & 8,7 & 5 & 21,7 & 2 & 8,7 & 14 & 60,9 \\
Urbana & 11 & 47,8 & 1 & 4,3 & 4 & 17,4 & 7 & 30,4 \\
\hline
\end{tabular}

A maioria dos participantes obteve pontuações entre 20 a 42 na escala da frequência alimentar. A diferença entre a pontuação para cada sexo não foi significativa (Tabela 4).

Tabela 4: Frequência alimentar dos participantes da pesquisa, munícipes de Gramados dos Loureiros, RS, janeiro a março de 2020.

\begin{tabular}{lccccc}
\hline & \multicolumn{2}{c}{ Feminino } & \multicolumn{2}{c}{ Masculino } & \\
Escore & Frequência & Percentual & Frequência & Percentual & Significância \\
\hline Até 28 & 7 & 28,0 & 6 & 28,6 & \\
29 a 42 & 16 & 64,0 & 14 & 66,7 & $\mathrm{p}=0,90$ \\
43 ou mais & 2 & 8,0 & 1 & 4,8 & \\
Total & $\mathbf{2 5}$ & & $\mathbf{2 1}$ & & \\
\hline
\end{tabular}

Os participantes relataram consumir mais alimentos naturais do que os industrializados. A frequência alimentar semanal para os grupos de saladas cruas, frutas frestas e feijão ultrapassou quatro vezes. A menor frequência semanal foi verificada para o grupo de refrigerantes. As frequências médias encontradas diferiram significativamente entre os grupos de alimentos (Figura 1). 


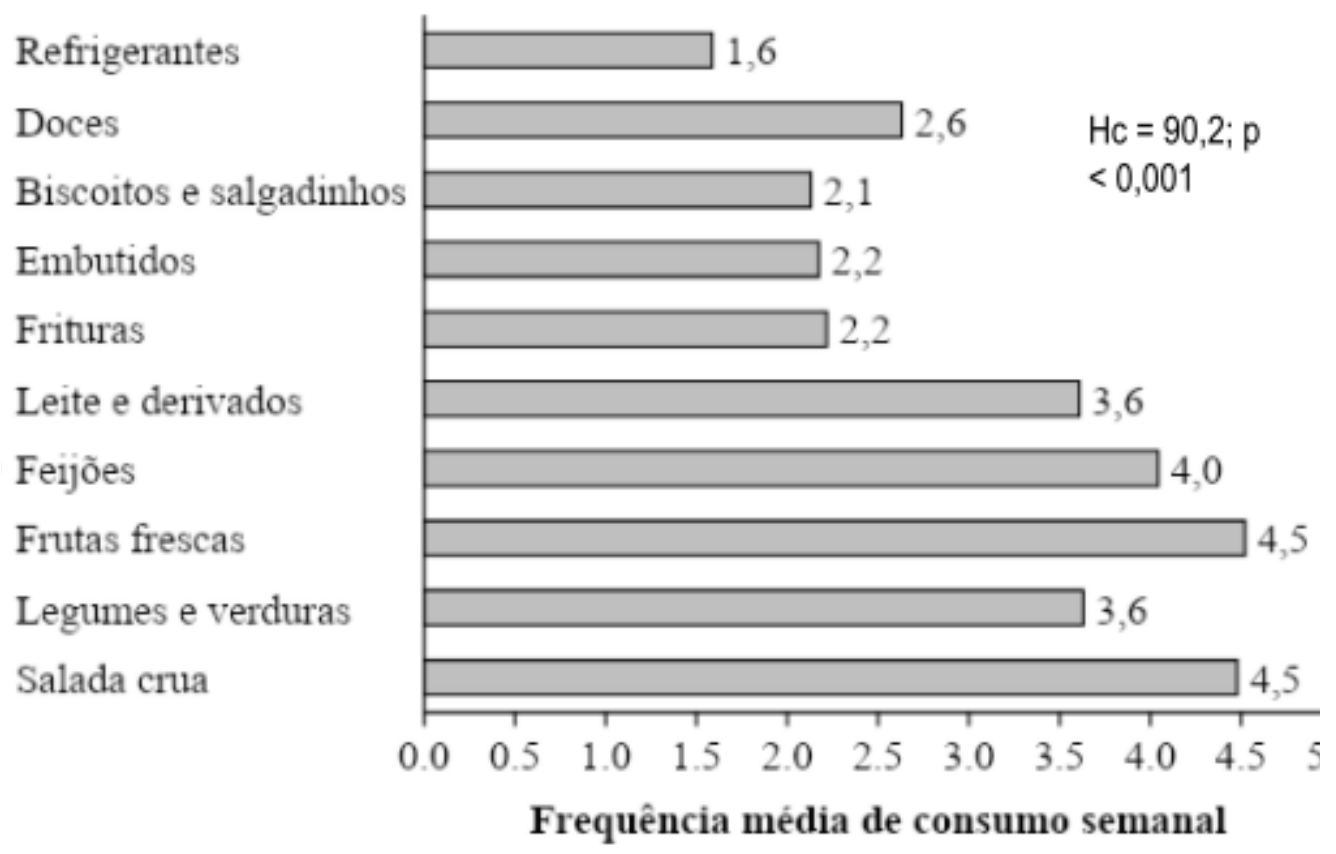

Figura 1: Recordatório do consumo alimentar dos participantes da pesquisa, munícipes de Gramado dos Loureiros, janeiro a março de 2020.

Dentre os participantes, a maioria já consultou ou teve um membro da família atendido por um profissional Nutricionista. Os participantes acreditam que o profissional Nutricionista agrega qualidade ao serviço de saúde no município. A maioria acha importante ter acesso e acompanhamento do profissional nas fases gestacional, da infância ou envelhecimento. Mais da metade dos entrevistados relataram ter alguma doença relacionada à má alimentação, como diabetes ou hipertensão. Os participantes acreditam que para uma boa alimentação é preciso ter uma alimentação equilibrada e variada (Quadro 1).

Quadro 1: Percepção dos participantes da pesquisa quanto a atuação do profissional Nutricionista. Município de Gramado dos Loureiros, RS, janeiro a março de 2020.

\begin{tabular}{|ll|}
\hline & Alimentação variada (comer de tudo): $26 \%$ \\
Percepção dos participantes quanto a uma & Alimentação equilibrada: $41 \%$ \\
boa alimentação. & Fazer seis refeições ao dia: 17\% \\
& Não consumir produtos industrializados: $7 \%$ \\
& Carnes sem gordura: $9 \%$ \\
\hline $\begin{array}{l}\text { Consulta do participante ou familiar a um } \\
\text { profissional Nutricionista. }\end{array}$ & Sim: $82 \%$ \\
\hline Adesão às recomendações do profissional $18 \%$ \\
Nutricionista & Sim (reeducação alimentar/dietas da moda): $80 \%$ \\
\hline Modificação da dieta pelos participantes ou ou & Sam: $20 \%$ \\
familiares. & Não: $18 \%$ \\
\hline
\end{tabular}




\begin{tabular}{|c|c|}
\hline $\begin{array}{l}\text { Adesão de tratamento pelos participantes } \\
\text { ou algum familiar por doenças } \\
\text { relacionadas à má alimentação. }\end{array}$ & $\begin{array}{l}\text { Sim: } 56 \% \\
\text { Não: } 44 \%\end{array}$ \\
\hline $\begin{array}{l}\text { Conhecimento pelo participante de um } \\
\text { profissional Nutricionista atuando no } \\
\text { município ou região. }\end{array}$ & $\begin{array}{l}\text { Sim: } 93 \% \\
\text { Não: } 7 \%\end{array}$ \\
\hline $\begin{array}{l}\text { Percepção do participante referente ao } \\
\text { agregamento de qualidade pelo } \\
\text { Nutricionista ao serviço de saúde no } \\
\text { município. }\end{array}$ & $\begin{array}{l}\text { Concordo: } 85 \% \\
\text { Discordo: } 9 \% \\
\text { Não tenho conhecimento: } 6 \%\end{array}$ \\
\hline $\begin{array}{l}\text { Sistema de atendimento do profissional } \\
\text { Nutricionista no município. }\end{array}$ & Público: $100 \%$ \\
\hline $\begin{array}{l}\text { Percepção do participante sobre } 0 \\
\text { acompanhamento nutricional nas fases de } \\
\text { gestação, infância ou envelhecimento. }\end{array}$ & $\begin{array}{l}\text { Sim, importante: } 91 \% \\
\text { Não: } 9 \%\end{array}$ \\
\hline $\begin{array}{l}\text { Percepção do participante sobre a } \\
\text { eficiência de um tratamento. }\end{array}$ & $\begin{array}{l}\text { Nutricionista: } 58 \% \\
\text { Medicamento: } 27 \% \\
\text { Depende de cada caso: } 15 \%\end{array}$ \\
\hline
\end{tabular}

\section{DISCUSSÃO}

O perfil da amostra abordada está de acordo com a realidade regional de pequenos municípios do sul do Brasil. Gramado dos Loureiros é um município com a sua economia baseada na agricultura e em pequenas propriedades ${ }^{11}$, portanto, é esperado que a população rural se equipare em número com a população urbana e que a ocupação urbana se concentre no comércio e na prestação de serviços.

Os achados apontam índice elevado de insegurança Alimentar e Nutricional (IAN) nas famílias do município. Esse resultado ratifica a situação de vulnerabilidade social dessas famílias e o risco de possíveis prejuízos para o crescimento e 0 desenvolvimento de suas crianças em função de uma alimentação inadequada ${ }^{8}$. A realidade encontrada ${ }^{11}$ representa um alerta para autoridades de saúde de outros municípios brasileiros com realidades sociais semelhantes.

A insegurança alimentar predominante entre os participantes do sexo masculino pode ser explicado pelo fato de que alguns maridos e/ou chefes de família do gênero masculino sentem insegurança em relação ao valor mensal salarial e a necessidade de quitação de suas dívidas. Por outro lado, o fato de que mais da metade dos entrevistados que se identificaram como chefes de famílias serem mulheres corrobora o estudo da PNAD no Brasil, onde as famílias chefiadas por mulheres mais que dobraram entre 2001 e $2015^{16}$. Esse achado reitera os avanços das políticas públicas das últimas duas décadas quanto ao empoderamento do sexo feminino na sociedade, contudo ressalta que a percepção difere entre homens e mulheres quando se trata da insegurança alimentar da família.

Identificou-se que $60 \%$ dos entrevistados trabalham fora de casa. Conforme estudo realizado no Estado do Rio de Janeiro no ano 2016, 57,6\% dos indivíduos que trabalham fora de casa optam por alimentos mais práticos 
e acessíveis em virtude dos custos e da falta de tempo. Isso reflete nos hábitos e nas preferências alimentares da população. Costumes podem refletir negativamente na saúde do indivíduo bem como na questão da segurança alimentar da família ${ }^{17}$.

A complexidade de se habituar a um consumo de alimentos saudáveis ${ }^{18}$, acessíveis e rápidos está interligada ao acesso, renda familiar e principalmente disponibilidade de tempo agregado ${ }^{19}$. 0 momento da refeição é um dos principais elos entre a família e 0 ato de nutrir o corpo ${ }^{20}$. Percebe-se na amostra avaliada uma dificuldade por uma alimentação no ambiente domiciliar, em contrapartida à preferência por alimentos in natura ou minimamente processados.

A maioria dos participantes obteve pontuações entre 20 a 42 na escala da frequência alimentar. Foi possível perceber que a pontuação é pouco satisfatória. Ainda, 28\% apresentaram pontuações até 28 na escala de frequência alimentar, pontuação que requer atenção e um melhor estilo de vida segundo o mesmo documento. Para os participantes com escore de 43 ou mais, a orientação é manter-se desta forma ativa, pois está no caminho para uma vida saudável21.

Os resultados indicam que a população de Gramado dos Loureiros possui hábitos alimentares favoráveis à saúde, com alta frequência de consumo de legumes, verduras e frutas e baixo consumo de alimentos industrializados. Tendo em vista o pequeno porte do município e a participação de agricultores dentre os participantes, os quais produzem o seu próprio alimento, explica-se o padrão encontrado. Os resultados também apontam para uma maior preferência por alimentos de boa origem nutricional, como o arroz, feijão, carnes e saladas, o que indica uma boa qualidade alimentar e nutricional da população gramadense.

Entrevistados da zona rural apresentaram um maior índice de segurança alimentar $(60,9)$, em relação à zona urbana $(30,4)$. Foi possível perceber que os participantes da zona rural relataram consumir mais alimentos naturais do que os industrializados, visto que se alimentam, na maioria das vezes, de alimentos da própria produção ou comprados de produtores locais. Os grupos alimentares mais frequentemente consumidos por semana foram os legumes, frutas, verduras as, leite e derivados. Entende-se que este consumo está associado ao acesso favorecido pela autoprodução de alimentos 22,23 .

Os resultados encontrados indicam uma alimentação a partir de produtos in natura ou minimamente processados, produzidos no próprio município. Relatório da FAO ${ }^{24}$ aponta que, entre os anos de 2010 e 2012, 19,1\% da população mundial sofria privação de acesso aos alimentos, sendo um percentual maior em países de baixa renda. Segundo 0 Ministério de desenvolvimento social (2016) (MDS), no período de 2002 a 2014, o Brasil reduziu em 82\% o número de brasileiros que se alimentavam mal ou em quantidade insuficiente. Contemporaneamente existem programas e/ou organizações que incentivam a produção familiar e a segurança alimentar dos brasileiros das zonas rurais e urbanas que, até então, se encontravam sob risco alimentar e social ${ }^{25}$. 
Uma alimentação saudável pode ser traduzida na garantia e condições de acesso a alimentos básicos de qualidade a todos, em quantidade suficiente, de modo permanente e sem comprometer o acesso a outras necessidades essenciais, com base em práticas alimentares saudáveis, contribuindo, assim, para uma existência digna, considerando todos os aspectos que são importantes para o desenvolvimento completo de um indivíduo saudáve ${ }^{24}$. Neste sentido, pode-se perceber que as famílias cujos chefes de família participaram da pesquisa, têm acesso a uma alimentação adequada, mas de forma intermitente. Os relatos dos participantes indicam que estes não podem garantir o acesso contínuo de alimentos sem comprometer as demais necessidades básicas.

A maioria dos participantes reconhece a importância do acompanhamento nutricional na infância, no envelhecimento ou na fase gestacional. 0 acompanhamento nutricional na fase da gestação além de ser importante para as mudanças de hábitos alimentares, ainda influencia na assistência nutricional, no controle de ganho de peso e sinais/sintomas relacionados à gravide ${ }^{26}$. Os relatos indicam um o cuidado maior quando em gestação ou em relação à alimentação dos filhos, onde é priorizado o consumo de verduras e legumes. Esse cuidado diz respeito ao conhecimento acerca da importância do alimento e seus impactos em cada indivíduo e/ou fase de vida, podendo assim informar sobre a importância dada à alimentação. 0 processo de nutrição (ingestão, digestão, absorção, metabolismo e excreção) é um dos fatores para manter as funções do organismo em dia em todas as fases da vida. Porém, é no envelhecimento que as maiores dificuldades começam aparecer, como a dificuldade de mastigação, por exemplo27. Uma boa alimentação tem relação positiva sobre a prevenção e o tratamento de doenças28. Mudanças no estilo de vida voltadas para um plano alimentar, não são imediatas.

O SUS é a única forma de acesso ao atendimento de um profissional Nutricionista no município de Gramados dos Loureiros. A Estratégia Saúde da Família está diretamente ligada ao bem estar da população e o Nutricionista é o profissional plenamente capacitado para atuar junto na atenção básica em saúde, visto que a alimentação adequada está associada à prevenção de doenças e que o profissional pode contribuir para a integralidade das ações de saúde².

A maioria dos participantes ou algum membro da família já foram atendidos por um Nutricionista. Doenças associadas à alimentação e melhores estilos de vida foram relatados como motivos. Um total de $80 \%$ relatou que aderiu à dieta prescrita pelo profissional. Hábitos alimentares prévios são os que mais interferem na adesão à dieta pelo paciente seguindo de aspectos emocionais e apoio familiar e/ou social ${ }^{29}$. Nesse sentido, os achados apontam para um reconhecimento positivo da população do município acerca do trabalho do profissional Nutricionista. A adesão ou não adesão tem impactos sobre a morbimortalidade dos indivíduos, principalmente aqueles acometidos por doenças crônicas $^{30}$. 


\section{CONSIDERAÇÕES FINAIS}

As famílias avaliadas apresentaram alta prevalência de insegurança alimentar e nutricional, mesmo sendo produtores de seu próprio alimento. Os resultados mostraram que a maioria das famílias possui uma alimentação adequada em quantidade e qualidade, entretanto, tem receio de que a situação possa mudar em um futuro próximo.

Os participantes relatam consumir todos os tipos de alimentos, incluindo saladas, frutas, verduras, leites e derivados. Os resultados indicam que o serviço de Nutrição existe no município, está disponível e é reconhecido pela população. Os participantes reconhecem o trabalho do profissional Nutricionista no contexto dos serviços de saúde, contudo buscam atendimento em situações de agravos ou de risco em que se encontram.

O estudo apresenta como limitação o fato de ter sido desenvolvido em apenas um município o que, evidentemente, aponta para particularidades. Recomendam-se estudos adicionais, tanto em outros municípios com realidades diferentes, como em momentos diferentes, dado o caráter dinâmico do tema abordado.

\section{REFERÊNCIAS}

1. Figueroa-Pedraza D, Alves-Bezerra T, Cerqueira ACD. R. (In)-Segurança alimentar de famílias residentes em um município do interior da Paraíba, Brasil. Revista de Salud Publica. 2017; 19 (5): 649-656.

2. Brasil. Lei n 11.346, de 15 de setembro de 2006: Lei de segurança alimentar e nutricional. Brasília: Presidência da República, 2006. [citado em 04 set 2019].

3. Rigon AS, Schimidt T, Bógus CM. Desafios da nutrição no Sistema Único de Saúde para construção da interface entre a saúde e a segurança alimentar e nutricional. Cadernos de Saúde Pública. 2016: 32(3):e00164514.

4. IBGE. Pesquisa Suplementar de Segurança Alimentar PNAD 2013. Rio de Janeiro: Instituto Brasileiro de Geografia e Estatística, 2014.

5. FAO. El estado de la seguridad alimentaria y la nutrición en el mundo. Fomentando la resiliencia climática en aras de la seguridad alimentaria y la nutrición. Roma: World Food and Agriculture, 2018.

6. Junior RRF, Santa Rita LP. Impactos da Covid-19 na Economia: limites, desafios e políticas. Cadernos de Prospecção - Salvador. 2020; 13(2):459-476.

7. Izar MCO, Fonseca FAH, Xavier HT. Obesidade e deslipidemia-metas de redução; uso de dietas e medicamentos. Revista da Sociedade de Cardiologia. 2019; 29(2): 148-154.

8. Fernandes ETP, Souza MNL, Rodrigues SM. Práticas de grupo do Núcleo de Apoio à Saúde da Família: perspectiva do usuário. Physis, Rio de Janeiro. 2019; 29(1):e290115. 
9. Torres GMC, Figueiredo IDT, Cândido JAB, Pinto AGA, Morais APP, Araújo MFM, Almeida MI. Comunicação terapêutica na interação profissional de saúde e hipertensão na estratégia saúde da família. Revista Gaúcha de Enfermagem. 2018; 38(4): 1983-1447.

10. Creswell JW. Projeto de Pesquisa: métodos qualitativo, quantitativo e misto. 3a ed. Porto Alegre: Artmed, 2010, 296 p.

11. IBGE. Ibge cidades: Gramado dos Loureiros. Instituto Brasileiro de Geografia e Estatística, 2019.

12. Pederneiras MMM, Lopes JEG, Ribeiro Filho JF, Feitosa MGG. Exame Nacional de Desempenho dos Estudantes na visão de líderes formais. Ensaio: Avaliação e Políticas Públicas em Educação. 2011; 19(71): 381-400.

13. Jannuzzi PM, Cunha JVQ, Pinto AR. Escala Brasileira de Insegurança Alimentar - EBIA: análise psicométrica de uma dimensão de Segurança Alimentar e Nutricional. Brasília 2014.

14. Esperandio N, Morais DC, Priore SE. Escalas de percepção da insegurança alimentar validadas: a experiência dos países da América Latina e Caribe. Ciência e Saúde Coletiva. 2016; 23(2): 449-462.

15. Machado CH, Carmo AS, Horta PM, Lopes ACS, Santos LC. Efetividade de uma intervenção nutricional associada à prática de atividade física. Cadernos Saúde Coletiva. 2013; 21(2): 148-153.

16. Cavenaghi S, Alvez JED. Mulheres chefes de família no Brasil: avanços e desafios. Estudo sobre seguros. Rio de Janeiro: ENS-CPES, 2018, 120 p.

17. Lindemann IL, Oliveira RR, Mendoza-Sassi RA. Dificuldades para alimentação saudável entre usuários da atenção básica em saúde e fatores associados. Ciência e Saúde Coletiva. 2016; 21(2): 599-610.

18. Aires JS, Martins MC, Joventino ES, Ximenes LB. (In) Segurança alimentar em famílias de pré-escolares de uma zona rural do Ceará. Acta Paulista de Enfermagem. 2012; 25(1): 102-108.

19. Rendeiro LC, Ferreira CR, Souza AAR, Oliveira DA, Della Noce RR. Consumo alimentar e adequação nutricional de adultos com obesidade. RBONE - Revista Brasileira de Obesidade, Nutrição e Emagrecimento. 2019; 12(76): 996-1008.

20. Defante LR, Nascimento LDO, Lima-Filho DO. Comportamento de consumo de alimentos de famílias de baixa renda de pequenas cidades brasileiras: o caso de Mato Grosso do Sul. Interações (Campo Grande). 2015; 16(2): 265-276.

21. Brasil. Guia alimentar para a população Brasileira. Brasília: Ministério da saúde, 2014, 158 p.

22. Fonseca AB, Souza TSN, Frozi DS, Pereira RA. Modernidade alimentar e consumo de alimentos: contribuições sócio-antropológicas para a pesquisa em nutrição. Ciência e saúde Coletiva. 2017; 16(9): 3853-3862.

23. Gramado dos Loureiros. História do município. Rio Grande do Sul, 2020. Disponível em: https://www.gramadodosloureiros.rs.gov.br/Sobre_o_munic\%C3\%ADpio. [citado em 04 set 2019]. 
24. FAO. Fao statistical Yearbook 2013. Roma: World Food and Agriculture, 2013, 307 p.

25. Brasil. Um sistema público para garantir a alimentação adequada. Brasília: Ministério do Desenvolvimento Social e Combate à Fome (MDS), 2016.

26. Barbosa AM, Araújo LSA, Barbosa VMO, Guerra FAM. Percepções maternas sobre a assistência nutricional no acompanhamento interdisciplinar do pré-natal e puerpério. Tempus, Acta de saúde coletiva. 2018; 11(2): 9-24.

27. Andrade DS, Ferreira JS, Souza US, Ramos MSX. Percepção acerca do envelhecimento saudável e das questões raciais. Revista de enfermagem UFPE. 2019; 13(1): 281-287.

28. Santos SLF, Barros KBNT, Prado RMS. Interações entre fármacos e nutrientes: ocorrência e manejo clínico. Revista de Ciências Médicas e Biológicas (Impr.). 2018; 17(1): 65-70.

29. Pereira J, Frizon E. Adesão ao tratamento nutricional de portadores de diabetes mellitus tipo 2: Uma revisão bibliográfica. Revista da Associação Brasileira de Nutrição. 2017; 8(2): 58-66.

30. Estrela KCA, Alves ACDC, Gomes TT, Isosaki M. Adesão às orientações nutricionais: uma revisão de literatura. Demetra, Alimentação, Nutrição e saúde. 2017; 12(1): 249-274. 